\title{
Mulheres Vítimas de Violência Doméstica: uma Abordagem no Núcleo da Delegacia em Atendimento à Mulher de um Município do Estado da Bahia
}

\author{
Women Victims of Domestic Violence: An Approach In The Women \\ Police Station In a Municipality of Bahia State
}

\author{
ZILMA DOS REIS MENDES ${ }^{1}$ \\ WANDERLEY DE ALCÂNTARA NOGUEIRA ${ }^{1}$ \\ JUCINEIAFAGUNDES DIAS ${ }^{1}$ \\ CRIS DE ASSIS SOUZA ${ }^{1}$ \\ MICHELE SANTANA PACHECO DE ALMEIDA ${ }^{2}$ \\ HELSON FREITAS DA SILVEIRA ${ }^{3}$ \\ HOWARD LOPES RIBEIRO JUNIOR ${ }^{4}$
}

\section{RESUMO}

Objetivo: Buscou-se realizar um levantamento do perfil das vítimas de violência doméstica, atendidas em um núcleo de assistência à mulher no município de São Francisco do Conde, estado da Bahia. Material e métodos: Trata-se de um estudo do tipo quali-quantitativo com base em 67 registros de boletins de ocorrência de mulheres vitimas de violência doméstica que deram entrada no Núcleo da Delegacia Especializada em Atendimento à Mulher (NUDEAM), no período de 2012 a 2014. Resultados: Foi possível identificar que houve um predomínio de registros nos anos de 2012 (29/67; 43,3\%) e 2014 (30/67, 44,8\%) com descrição de vítimas com idade entre $19-39$ anos $(40 / 67 ; 59,7 \%)$, com etnia predominante negra $(15 / 67 ; 22,4 \%)$, com ensino fundamental incompleto (16/ $67 ; 23,9 \%$ ), com renda familiar de um salário mínimo adicionado de benefício social $(22 / 67 ; 33 \%)$ e, a maioria destas, possuindo entre 1 a 2 filhos $(29 / 67 ; 43,3 \%)$. Quanto ao tipo de violência registrada no boletim de ocorrência, destacouse os casos de agressão física adicionada de três ou mais outros tipos de violência $(16 / 67 ; 23,9 \%$ ) e da medida protetiva de Orientação sobre a Lei Maria da Penha à vítima (18/67; 26,9). Conclusão: Houve um aumento no número de casos de vítimas de violência doméstica atendidas no NUDEAM entre os anos de 2012 e 2014, compreendendo mulheres jovens, negras, com baixa escolaridade e renda familiar, com 1 a 2 dois filhos e submetidas à violência física adicionada de três ou mais tipos de violência por parte do agressor, sendo este, na maioria dos casos, o próprio companheiro.

DESCRITORES

Violência. Violência doméstica. Saúde Pública.

\begin{abstract}
Objective: We aimed to conduct a survey on the profile of victims of domestic violence at a women care center in São Francisco do Conde, Bahia. Material and methods: This was a qualitative and quantitative study analyzing 67 reports of of domestic violence against women. he reports were filed at the Center for Specialized Police for Assistance to Women (NUDEAM) from 2012 to 2014. Results: There was a predominance of reports in the years $2012(29 / 67 ; 43.3 \%)$ and $2014(30 / 67,44.8 \%)$ with description of victims aged 19-39 years $(40 / 67 ; 59.7 \%)$; black $(15 / 67 ; 22.4 \%)$; with incomplete primary education $(16 / 67 ; 23.9 \%)$; family income of a minimum wage plus social benefits (22/67; 33\%); and having about 1 to 2 children $(29 / 67 ; 43.3 \%)$. Regarding the type of violence recorded in the police report, there were cases of physical aggression added of three or more other types of violence (16/ $67 ; 23.9 \%)$; and the protective guidance measure about Maria da Penha Law $(18 / 67 ; 26.9)$. Conclusion: There was an increase in the number of cases of domestic violence filed at NUDEAM between the years 2012 and 2014, including young women, black, with low education and family income, with 1 to 2 children and subjected to physical violence added to three or more types of violence by the perpetrator, in most cases, the partner himself.
\end{abstract}

DESCRIPTORS

Violence. Domestic violence. Public Health

1 Estudante de Pós-graduação em Especialização em Gestão de Saúde. Programa Nacional de Administração Pública, Universidade Aberta do Brasil. Universidade da Integração Internacional da Lusofonia Afro-Brasileira, Campus de São Francisco do Conde, Bahia, Brasil.

2 Tutora do curso de Especialização em Gestão de Saúde. Programa Nacional de Administração Pública, Universidade Aberta do Brasil. Universidade da Integração Internacional da Lusofonia Afro-Brasileira, Campus de São Francisco do Conde, Bahia, Brasil.

3 Médico Veterinário. Mestrando em Ciências Morfofuncionais pela Universidade Federal do Ceará. Tutor do curso de Especialização em Gestão de Saúde. Programa Nacional de Administração Pública, Universidade Aberta do Brasil. Universidade da Integração Internacional da Lusofonia AfroBrasileira, Campus de São Francisco do Conde, Bahia, Brasil.

4 Doutorando em Ciências Médicas pela Universidade Federal do Ceará. Professor-Pesquisador II do curso de Especialização em Gestão de Saúde. Programa Nacional de Administração Pública, Universidade Aberta do Brasil. Universidade da Integração Internacional da Lusofonia Afro-Brasileira, Campus de São Francisco do Conde, Bahia, Brasil. 
$\mathrm{A}$ violência doméstica é um ato de agressão, cometido por marido ou companheiros, familiares e/ou indivíduos desconhecidos, podendo incluir diferentes manifestações, sendo considerado hoje um importante problema de saúde pública ${ }^{1}$. A violência doméstica é um mal que aflige a sociedade e é vista como uma violação de direitos humanos, pois merece rigorosa punição para os agressores, já que antes não existiam leis e órgãos de amparo às vítimas de violência ${ }^{2}$.

Historicamente, a legislação brasileira, ainda no século XIX, não reconhecia os casos de violência contra a mulher como crime, portanto não havia punição para os seus agressores. Atualmente a violência doméstica ainda faz parte da realidade de muitas mulheres brasileiras e, devido à sua gravidade, a temática tornou-se uma questão de saúde pública que vem ganhando grandes repercussões no cenário nacional ${ }^{3,4}$. Diante desta realidade, criou-se a Lei $\mathrm{n}^{\mathrm{o}}$ 10.778, promulgada em 24 de novembro de 2003 , correspondendo a um importante passo no combate ao crime de violência contra a mulher, na qual obriga a todos os serviços de saúde, as entidades que prestam serviço às vítimas seja na esfera pública ou privada, notificar os casos suspeitos ou confirmados de violência contra a mulher 5 .

Outra conquista importante foi a criação da Lei $\mathrm{n}^{\mathrm{o}} 11.340$, de 07 de agosto de 2006, comumente conhecida por Lei Maria da Penha, como forma de coibir os crimes de violência doméstica e punir com mais rigor os agressores ${ }^{5}$. Essa Lei é uma importante conquista no enfrentamento da violência doméstica, pois prevê punição aos agressores envolvidos nos casos de violência, e garante "à mulher o direito à sua integridade física, psíquica, sexual e moral"'. Após entrar em vigor, esta lei impõe que o agressor pode ser preso em flagrante, ou ter sua prisão decretada em qualquer situação de ameaça ou violação de direito a mulher ${ }^{7}$. Todas essas ações são asseguradas através das delegacias especializadas em atendimento à mulher (DEAM), implantadas para atender essas necessidades ${ }^{8}$.

Segundo levantamento realizado ${ }^{7}$ a violência doméstica vem crescendo assustadoramente no país. No Brasil, uma mulher sofre algum tipo de violência a cada 15 segundos, tendo, na maioria dos casos, como principal agressor, o próprio companheiro, muitas vezes sobre o efeito de drogas lícitas ou ilícitas ${ }^{5}$. Entre os tipos de violências praticadas, a de maior incidência é a agressão física, e, vale acrescentar que em termos de homicídios, o Brasil ocupa a sétima posição a nível mundial, com uma taxa de 4,4 homicídios em cada 100 mil mulheres ${ }^{9}$. Na Região Nordeste, em especial, no Estado da Bahia, vê-se que este ocupa a terceira posição no ranking nacional, com $14,15 \%$ de registros de casos de violência doméstica, possuindo, dentre as ocorrências, a violência física com maior prevalência e os companheiros/maridos como principais perpetradores ${ }^{10}$.

Especificamente no município de São Francisco do Conde, no ano de 2012, observou-se um importante crescimento no número de casos de violência doméstica, com características semelhantes às relatadas no estado da Bahia, gerando um importante impacto na saúde do referido município ${ }^{11}$. No entanto, a identificação de casos de violência doméstica muitas vezes se torna difícil por que esse é um fenômeno que ocorre num ambiente privado do lar e conta com o silêncio das vítimas ${ }^{11}$.

Frente a este contexto, faz-se necessário a investigação desse fenômeno, pois a violência sofrida por estas mulheres pode interferir em diversos aspectos de suas vidas, desencadeando diversos problemas para a saúde das vítimas criando demandas para diversos setores sociais inclusive para o setor saúde ${ }^{11}$. Assim, frente ao exposto, o presente estudo buscou avaliar o perfil de vítimas de violência doméstica atendidas pelo núcleo da delegacia especializada em atendimento à mulher (NUDEAM) no município de São Francisco do Conde, na Bahia, no período de 2012 a 2014, a fim de gerar dados que contribuam para o desenvolvimento das políticas públicas do município através da tomada de novas medidas de prevenção e promoção à saúde da mulher sanfranciscana.

\section{MATERIAISE MÉTODOS}

Trata-se de um estudo do tipo documental, descritivo e de abordagem quantitativa tendo como unidade de análise o Núcleo da Delegacia Especializada em Atendimento à Mulher (NUDEAM), realizado a partir de dados secundários referentes ao período de 2012 a 2014. O NUDEAM está situado no município de São Francisco do Conde, Bahia. Este núcleo é um serviço credenciado pela Secretária de Desenvolvimento Social (SEDES) criado como subsídio para as mulheres vitimadas de todo tipo de violência, visto que, o município tem uma população inferior (38.838 habitantes $)^{20}$ ao número aceitável para a implantação de uma Delegacia Especializada no Atendimento à Mulher (DEAM) ${ }^{10}$. Para isto, o NUDEAM embasa-se na Lei $\mathrm{n}^{\circ} 11.340$ - Lei Maria da Penha - a qual dispõem de medidas especiais em casos de violências contra a mulher, na tentativa de garantir que o direito feminino seja efetivado.

Os dados das vítimas foram obtidos através dos registros de boletins de ocorrência de mulheres 
vitimas de violência doméstica atendidas no NUDEAM, em São Francisco do Conde, no período de 2012 a 2014, quando deu-se inicio ao primeiro ano de atividades do referido núcleo.

Constituíram-se como variáveis e indicadores avaliados neste estudo: idade, tipo de violência, tipo de intimidação por parte do agressor, classe social, escolaridade, profissão, naturalidade, estado civil, raça/ etnia, medida protetiva aplicada e o tipo de relação da vítima com o agressor. Foram excluídos deste estudo os registros de mulheres sem relação com a violência doméstica no período estabelecido na pesquisa.

Os dados obtidos a partir das análises das variáveis contidas no levantamento dos boletins de ocorrência das vítimas de violência doméstica no Núcleo da Delegacia Especializada em Atendimento a Mulheres submetidas a este estudo foram tabulados através de estatística analítico-descritiva, com auxílio do software Microsoft Office Excel 2010 (Microsoft Corporation, Redmond, WA).

A presente pesquisa foi aprovada pelo Comitê de Ética em Pesquisa da Universidade da Integração Internacional da Lusofonia Afro-Brasileira, sob o registro de $\mathrm{n}^{\circ} 866.696$ através do sistema da Plataforma Brasil.

\section{RESULTADOS}

Ao todo foram avaliados 67 registros de boletins de ocorrência de mulheres vítimas de violência doméstica atendidas no NUDEAM. A distribuição das variáveis sociodemográficas é apresentada na Tabela 1.

A distribuição das vítimas assistidas pelo NUDEAM, de acordo com o tipo de renda familiar, é apresentada na Figura 1.

Na Tabela 2, com base nos registros dos boletins das vítimas atendidas no NUDEAM, é apresentada a distribuição dos casos de acordo com o tipo de violência, de intimidação e de relação por parte do agressor, como também a medida protetiva aplicada para a vítima de violência doméstica.

\section{DISCUSSÃO}

Esse foi o primeiro estudo realizado na cidade de São Francisco do Conde, que procurou demonstrar o perfil das vítimas de violência doméstica atendida pelo núcleo da delegacia especializada em atendimento à mulher (NUDEAM) e sua repercussão na saúde do Município de São Francisco do Conde, no estado da Bahia.
Destaca-se que no relatório nacional brasileiro, que retrata o perfil da mulher brasileira, é informado que a cada 15 segundos uma mulher é agredida, totalizando, em 24 horas, um número de 5.760 mulheres espancadas no Brasil ${ }^{12}$. Assim, conforme os achados deste estudo, foi demonstrado que os anos de 2012 e 2013 apresentaram a maior taxa de casos de vítimas atendidas no NUDEAM. Um estudo realizado na Cidade de São Francisco do Conde em 2012 aponta que a violência doméstica vem crescendo no município, de certa forma invisível, rebatendo no setor saúde sobre diversas formas ${ }^{11}$. A identificação muitas das vezes se torna difícil por esse ser um fenômeno que ocorre em ambiente privado do lar e conta com o silêncio das vítimas ${ }^{11}$.

Com relação à idade das vítimas, os resultados mostram uma predominância de vítimas com idade entre 19-39 anos, com etnia predominante de negros, com ensino fundamental incompleto e com 1 ou 2 filhos (Tabela 1). Destaca-se que foram registrados atos de violência também contra as mulheres em diferentes faixas etárias, tais como em adolescentes e idosas (Tabela 1). Ressaltamos que os resultados desse estudo corroboram com outras pesquisas que apontam semelhanças no perfil das vítimas ${ }^{13}$, que demonstra que a variável idade apresentou semelhança entre duas faixas etárias da vida da mulher que sofreu violência sendo em grupos de 20 aos 29 anos e dos 30 aos 39 anos de idade. Esses resultados sugerem que a violência contra a mulher perpassa diferentes faixas etárias e momentos de vida da mulher ${ }^{13}$.

Quanto à etnia, os dados apresentados na presente pesquisa demonstraram uma predominância da etnia negra na população estudada. Este dado nos aponta para a relevância entre a categoria gênero e raça, já que a violência doméstica é definida pela organização dos Estados Americanos - OEA como: "qualquer ato ou conduta baseada no gênero, que cause morte, dano ou sofrimento físico, sexual ou psicológico à mulher, tanto na esfera pública como na esfera privada" 14 . Assim, é relevante destacar que a mulher brasileira negra sofre uma dupla violência, ou seja, a violência de gênero e a violência por ser negra, uma raça vista como inferior, status adquirido de um Brasil escravocrata.

Enfatizamos que, de acordo com a Lei Maria da Penha, configura-se violência doméstica e familiar contra a mulher, qualquer ação ou omissão baseada no gênero que lhe cause morte, lesão, sofrimento físico, sexual ou psicológico e dano moral ou patrimonial ${ }^{7}$. Neste sentido são formas de violência contra a mulher: a física, psicológica, sexual, patrimonial e a moral. Adicionalmente, a Vigilância em Saúde ampliou-se a definição de violência contra a mulher como sendo qualquer conduta, ativa ou passiva, seja de 
Tabela 1. Caracterização sóciodemográfica das vítimas atendidas no NUDEAM em São Francisco do Conde, Bahia, no período de 2012 a 2014.

\begin{tabular}{|c|c|}
\hline Variáveis & $\mathbf{N}^{0}(\%)$ \\
\hline $\mathrm{N}^{0}$ de casos & 67 \\
\hline \multicolumn{2}{|l|}{ Estratificação de casos / Ano } \\
\hline 2012 & $29(43,3)$ \\
\hline 2013 & $08(11,9)$ \\
\hline 2014 & $30(44,8)$ \\
\hline Idade ${ }^{a}$ & $31,4(11-92)$ \\
\hline$<18$ anos & $08(11,9)$ \\
\hline $19-39$ anos & $40(59,7)$ \\
\hline $40-59$ anos & $13(19,4)$ \\
\hline 60 anos ou mais & $01(1,5)$ \\
\hline Desconhecido b & $05(7,5)$ \\
\hline \multicolumn{2}{|l|}{ Etnia } \\
\hline Negro & $15(22,4)$ \\
\hline Pardos & $12(17,9)$ \\
\hline Branco & $01(1,5)$ \\
\hline Desconhecido b & $39(58,2)$ \\
\hline \multicolumn{2}{|l|}{ Escolaridade } \\
\hline Ensino Fundamental Incompleto & $16(23,9)$ \\
\hline Ensino Fundamental Completo & $03(4,5)$ \\
\hline Ensino Médio Incompleto & $04(5,9)$ \\
\hline Ensino Médio Completo & $12(17,9)$ \\
\hline Ensino Superior Incompleto & $01(1,5)$ \\
\hline Ensino Superior Completo & $02(3,0)$ \\
\hline Desconhecido $^{\mathrm{b}}$ & $29(43,3)$ \\
\hline \multicolumn{2}{|l|}{ Profissão } \\
\hline Autônoma & $03(4,5)$ \\
\hline Estudante & $04(6,0)$ \\
\hline Doméstica & $04(6,0)$ \\
\hline Auxiliar de serviços gerais & $06(8,9)$ \\
\hline Desconhecido ${ }^{b}$ & $50(74,6)$ \\
\hline \multicolumn{2}{|l|}{ Filhos } \\
\hline Sem filhos & $11(16,4)$ \\
\hline $1-2$ filhos & $29(43,3)$ \\
\hline 3 ou mais filhos & $22(32,8)$ \\
\hline Desconhecido b & $05(7,5)$ \\
\hline \multicolumn{2}{|l|}{ Distribuição Geoqráfica } \\
\hline Caípe de Baixo e Caípe de Cima & $17(25,4)$ \\
\hline São Bento & $09(13,4)$ \\
\hline Muribeca & $08(11,9)$ \\
\hline Nova São Francisco & $08(11,9)$ \\
\hline Pitanqueira & $05(7,5)$ \\
\hline Centro & $05(7,5)$ \\
\hline Outros Distritos & $12(17,9)$ \\
\hline Outras cidades ${ }^{c}$ & $02(3,0)$ \\
\hline Desconhecido $^{\mathrm{b}}$ & $01(1,5)$ \\
\hline \multicolumn{2}{|c|}{$\begin{array}{l}\text { a Valor de Média e Mediana (Máximo - Mínimo). } \\
\text { b Valores não informados nos boletins de ocorrência. } \\
\text { c Indivíduos são naturais de outras cidades do estado da } \\
\text { Bahia. }\end{array}$} \\
\hline
\end{tabular}




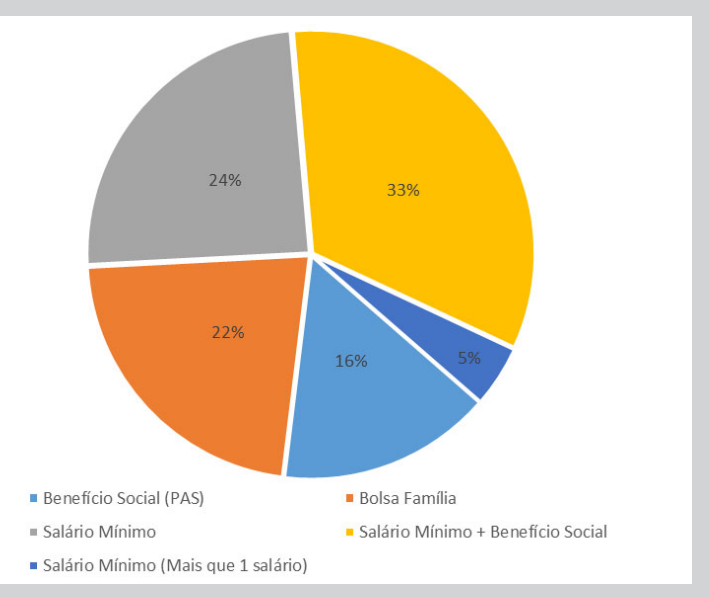

Figura 1: Estratificação das vítimas atendidas no NUDEAM em São Francisco do Conde, na Bahia, no período de 2012 a 2014 pela variável de fonte de renda familiar.

\begin{tabular}{|c|c|}
\hline $\begin{array}{l}\text { Variáveis associadas ao Registro do Boletim de } \\
\text { Ocorrência }\end{array}$ & $N(\%)$ \\
\hline \multicolumn{2}{|l|}{ Tipo de Violência registrada no Boletim de Ocorrência } \\
\hline Aaressão Física (sem definicão) & $10(14,9)$ \\
\hline $\begin{array}{l}\text { Agressão Física adicionada de } 1 \text { outro tipo de violência } \\
\text { (Moral, Patrimonial, Psicológica ou Sexual) }\end{array}$ & $13(19,4)$ \\
\hline $\begin{array}{l}\text { Agressão Física adicionada de } 3 \text { ou mais outros tipos de } \\
\text { violência (Moral, Patrimonial, Psicológica e Sexual) }\end{array}$ & $16(23,9)$ \\
\hline Agressão Moral & $11(16,4)$ \\
\hline Agressão Sexual & $02(3,0)$ \\
\hline Outros & $07(10,4)$ \\
\hline Desconhecido a & $08(11,9)$ \\
\hline \multicolumn{2}{|l|}{ Tipo de Intimidacão por parte do Agressor } \\
\hline Força Física (com ou sem o uso de Arma) & $10(14,9)$ \\
\hline Ameaça Verbal (Iniúria e Difamacão) & $07(10,5)$ \\
\hline Desconhecido a & $50(74,6)$ \\
\hline \multicolumn{2}{|l|}{ Medida Protetiva Aplicada } \\
\hline Orientação sobre a Lei Maria da Penha & $18(26,9)$ \\
\hline Encaminhamento para exame Pericial / Corpo de delito & $15(22,4)$ \\
\hline $\begin{array}{l}\text { Encaminhamento para o CRAS (Centro de Referência de } \\
\text { Assistência Social) }\end{array}$ & $04(6,0)$ \\
\hline $\begin{array}{l}\text { Registro de Boletim de Ocorrência sem indicativo de } \\
\text { medida protetiva }\end{array}$ & $09(13,4)$ \\
\hline Outros & $06(8,9)$ \\
\hline Desconhecido a & $15(22.4)$ \\
\hline \multicolumn{2}{|l|}{ Tipo de relação com o aqressor } \\
\hline Companheiro / Côniuge & $28(41,8)$ \\
\hline Ex-Companheiro / Côniuqe & $12(17,9)$ \\
\hline Parente Familiar (Pai, Irmão e Genro) & $08(11,9)$ \\
\hline Vizinho & $03(4,5)$ \\
\hline Outros & $11(16,4)$ \\
\hline Desconhecido a & $05(7,5)$ \\
\hline Total & 67 \\
\hline
\end{tabular}

a Valores não informados nos boletins de ocorrência. 
discriminação, agressão ou coerção, podendo ser motivada pela vítima se tratar de uma mulher, ocasionando danos a ela, seja por sofrimento físico, sexual, psicológico, morte, constrangimento ou limitação ${ }^{15}$.

Neste sentido, quanto ao tipo de violência registrada no boletim de ocorrência, houve predomínio da variável de agressão física adicionada de três ou mais outros tipos de violência (Moral, Patrimonial, Psicológica e Sexual) seguida de agressão física adicionada de outro tipo de violência (Moral, Patrimonial, Psicológica ou Sexual). Adicionalmente, observou-se que, com relação ao tipo de intimidação por parte do Agressor, houve predomínio da Força Física (com ou sem o uso de Arma) em 14,9\% dos casos. Estudos apontam que dentre as formas de violência supracitadas a mais comum é a física, consistindo no ato de provocar lesões corporais possivelmente diagnosticáveis, tais como cutâneas, neurológicas, oculares e ósseas, provocadas por queimaduras, mordidas, tapas, espancamentos, ou qualquer ação que ponha em risco a integridade física da mulher ${ }^{12}$. Todas as formas de violência aqui elencadas são capazes de provocar vários prejuízos à vida das mulheres vitimadas, acarretando diversas sequelas que poderão repercutir em toda a sua vida.

Reforçamos que a violência contra a mulher vem sendo apontada nas últimas décadas como problema social de saúde pública, pois compromete a integridade física e psíquica da mulher, além de constituir uma violação aos direitos humanos ${ }^{5}$. A Organização Mundial da Saúde (OMS) relaciona essa violência à maior ocorrência de diversos problemas de saúde física, reprodutiva e mental, assim como ao maior uso de serviços de saúde por parte das mulheres ${ }^{16}$. Os resultados desse estudo apontam para um real comprometimento da saúde das mulheres o que podem vir a impactar na normalidade dos processos em saúde pública do município de São Francisco do Conde.

Com relação às Medidas Protetivas Aplicadas, predominou a medida de Orientação sobre a Lei Maria da Penha em 26,9\% dos casos, seguida de Encaminhamento para exame de Pericial / Corpo de delito em $22,4 \%$ dos casos avaliados. As medidas protetivas aplicadas apontam ainda para a necessidade de uma melhor implementação e aplicabilidade das políticas públicas existentes para as vítimas de violência doméstica $^{13}$. De acordo com levantamentos feitos por alguns autores, a política pública é entendida como as respostas do Estado às demandas sociais de interesse da coletividade ${ }^{17}$.

Vê-se que não basta somente a implementação de políticas públicas voltadas para a proteção e segurança de mulheres em situação de violência se em conjunto não existirem ações de caráter educativo e conscientizador para quem precisa do serviço como para quem o fornece ${ }^{13}$. É destacado ainda que as delegacias de atendimento à mulher são essenciais para a garantia dos Direitos Humanos e internos, mas não o suficiente para o alcance de respostas efetivas às demandas femininas ${ }^{17}$.

Quanto ao tipo de relação da vítima com o agressor, houve predomínio da relação Companheiro/ Cônjuge seguida de ex-Companheiro/ex-cônjuge em $41,8 \%$ e $17,9 \%$ dos casos avaliados. Frente a este dado, existe uma categoria de gênero como fundamental para a compreensão dos papéis atribuídos ao homem e à mulher na sociedade, papeis estes que nos leva a compreendermos a violência infligida contra a mulher no ambiente doméstico. Dessa forma, a violência doméstica e familiar é também uma violência de gênero, pois esta surgiu a partir da organização social de gênero que beneficia o masculino ${ }^{18}$.

Na presente pesquisa, houve um predomínio de 33,0\% da população estudada com o salário mínimo adicionado de benefício social como única renda familiar, seguida de famílias que recebem exclusivamente o Salário Mínimo como renda familiar em $24 \%$ dos casos. É importante ressaltar que o Município de São Francisco do Conde, de acordo com o último censo do IBGE, apresenta uma das maiores rendas per capita do País ${ }^{19}$. No entanto, o município ainda não conta com uma rede de proteção completa, ou seja, com ações integradas para proteção e acolhimento às mulheres vítimas de violência, garantido-as um atendimento integral tais como a existência de uma Delegacia da mulher, de um centro de referência da mulher em situação de violência e uma casa abrigo. Destacamos que a violência contra a mulher manifesta-se de diversas formas e em diferentes espaços na sociedade, não importando a classe social, idade, raça/etnia, cultura ou grau de desenvolvimento econômico ${ }^{20}$.

Com relação à escolaridade das vítimas, houve uma prevalência de vítimas com nível de escolaridade apenas do ensino fundamental incompleto. "Pessoas com baixa escolaridade tendem a se tornar mais expostas aos agravos sociais e de saúde, [...] torna-se menos favorecida de recursos materiais bem como de informações de seus direitos sociais"13.

Uma das limitações deste estudo foi ocasionada pela dificuldade na obtenção e compreensão dos dados dos boletins de ocorrência das vítimas atendidas no NUDEAM, visto que, atualmente, estes boletins de ocorrências ainda estão descritos em formulários de papel, não possuindo, portanto, um sistema informatizado de registros eletrônicos de ocorrência das vítimas. Este fato é observado quando são citadas variáveis com elevado percentual de dados desconhecidos, tais como nas variáveis de idade, tipo de violência, tipo de Intimidação por parte do Agressor, 
classe social, escolaridade, profissão, naturalidade, estado civil, raça/etnia, medida protetiva aplicada, tipo de relação com o agressor.

Sugere-se a realização de estudos futuros cujas linhas investigatórias procedam à padronização de métodos de ampliem a identificação das vítimas de violência doméstica atendidas no NUDEAM, objetivando favorecer meios para que haja mais investimento em políticas do combate a esse tipo de violência, bem como garantir a aplicabilidade das medidas já existentes para coibir os agressores, empoderando as vítimas e evitando que seus direitos sejam violados, além de minimizar o impacto destes agravos na saúde pública do Município de São Francisco do Conde no estado da Bahia.

Sabendo-se que a violência contra a mulher é um problema de Saúde Pública, vê-se como importante questão o fato com que as mulheres, que são agredidas muitas vezes, não procuram atendimento médico e, quando o fazem, não raramente, a agressão não é identificada, ou porque elas omitem o ocorrido ou porque os médicos estão desavisados sobre a prevalência da violência contra a mulher e simplesmente não a pesquisam ${ }^{21}$. Estudos mostram que os médicos não pesquisam suas pacientes para violência por treinamento insuficiente, curto tempo do atendimento ou por perceberem uma falta de intervenção efetiva ${ }^{22}$.

Assim, aumentar a consciência popular e dos profissionais do campo da saúde sobre a existência de um agravo que pode ser prevenido, ou utilizando-se de um conteúdo de informação compreensível e atrativo, como elemento de conscientização, é uma medida estratégica que o gestor da saúde deve assumir no fomento de novas e ativas ações de promoção da saúde no município sob sua gestão. Neste contexto, inferimos que a prevenção dos danos à saúde das vítimas apresentados nesta pesquisa podem ser realizadas através de ações e implementações de políticas públicas que incluam tanto o combate quanto a prevenção desse tipo de violência, em especial aplicando as medicadas

\section{REFERÊNCIAS}

1. Andrade CJM, Fonseca RMGS. Considerações sobre violência doméstica, gênero e o trabalho das equipes de saúde da família. Rev. Esc. Enferm. USP., 2008; 42(3); 591-95.

2. Oliveira ER. Violência Doméstica e Familiar Contra a Mulher: Um Cenário de Subjugação do Gênero Feminino. Revista do Laboratório de Estudos da Violência da UNESP/Marília, 2012; (9): 150-165.

3. Berz, DSS, Violência Contra a Mulher: O Perfil da Mulher Vítima de Violência Doméstica no Município de Taquara/ RS, 2011 a Agosto de 2012 [Monografia de Graduação]. Taquara: Faculdades Integradas de Taquara; 2012. $150 p$. para coibir e punir os agressores, com o comprometimento dos profissionais da saúde, considerando-se que a proteção à saúde seja uma demanda concreta, permitindo assim, que todos os casos de violência doméstica sejam notificados pelas unidades de saúde.

\section{CONCLUSÕES}

A partir da execução do presente estudo, pôdese concluir que houve um aumento no número de casos de vítimas de violência doméstica atendidas no Núcleo da Delegacia Especializada em Atendimento à Mulher localizada no Município de São Francisco do Conde, estado da Bahia, entre os anos de 2013 e 2014, compreendendo mulheres com prevalência de idade 1939 anos, com predominância de etnia negra, com escolaridade o nível de ensino fundamental incompleto, com o predomínio de um salário mínimo adicionado de benefício social como única renda familiar e com 1 a 2 dois filhos. Adicionalmente, observou-se uma maior prevalência de casos de violência física adicionada de três ou mais tipos de violência com o predomínio da força física por parte do agressor sendo este, na maioria dos casos, o próprio companheiro.

\section{AGRADECIMENTOS}

Agradecemos o apoio da Sra. Luciana Araújo, gestora responsável pelo núcleo da delegacia especializada em atendimento à mulher no município de São Francisco do Conde, no estado da Bahia, por permitir e disponibilizar o acesso da equipe executora da presente pesquisa aos registros das vítimas de violência doméstica atendidas no respectivo órgão. Este estudo foi apoiado e suportado pela Coordenação de Aperfeiçoamento de Pessoal de Nível Superior, CAPES, juntamente com a Universidade Aberta do Brasil -UAB.
4. Dossi AP, Saliba O, Garbin CAS, Garbi AJL. Perfi epidemiológico da violência física intrafamiliar: agressões denunciadas em um município do Estado de São Paulo, Brasil, entre 2001 e 2005. Cad. Saúde Pública, 2008; 24(8):1939-1952.

5. Gomes VR, Lima VLA, Silva AF, Sena LX, Santos ACB. Violência Contra a Mulher na Região Norte: A Versão da Mídia Impressa Paraense. Revista do Laboratório de Estudos da Violência da UNESP, 2014; (14):113-128.

6. Ferraz MIR, Lacerda, MR, Labrocini LM, Maftum MA, Raimundo $\mathrm{ML}$. O cuidado de enfermagem às vítimas de violência doméstica. Cogitare Enferm, 2009;14(4): 7559. 
7. Brasil, Governo Federal, Secretaria de Políticas para as Mulheres: Lei $n^{\circ} 11.340$ de 7 de agosto de 2006 Disponível em: http://www.planalto.gov.br/ccivil 03/ _ato2004-2006/2006/lei//11340.htm. Acesso em: $2 \overline{5}$ de abril de 2015.

8. Organização Dos Estados Americanos. Convenção interamericana para prevenir, punir e erradicar a violência contra a mulher. In: Comissão Interamericana de Direitos Humanos, Belém, 1994.

9. Waiselfisz JJ. Mapa da violência 2012 - Atualização: Homicídios de mulheres no Brasil. Instituto Sangari. São Paulo; 2012.

10. Brasil, Governo Federal, Secretaria de Políticas para as Mulheres: Balanço Semestral Janeiro à junho/2012, Disque 180. Disponível em: http://www.spm.gov.br/ sobre/publicacoes/publicacoes/2012/balancosemestral-ligue-180-2012. Acesso em: 20 de abril de 2015.

11. Gomes NP, Silveira YM, Normélia MFD, Paixão, GPN, Camargo CL, Gomes NR. Identificação da Violência na Relação Conjugal a partir da estratégia saúde da família. Artigo Original. Texto Contexto Enferm, 2013; 22(3): 789-96.

12. Fonseca $L$, Violência doméstica contra a mulher e suas consequências psicológicas - Salvador-Ba, [Monografia de Graduação]. Salvador: Fundação Bahiana para o Desenvolvimento das Ciências; 2006. 21p.

13. Albuquerque C, Silva E, Azevedo F. Violência doméstica: características sociodemográficas de mulheres cadastradas em uma Unidade de Saúde da Família. Revista eletrônica de enfermagem 2013;15(2):382-390.

14. Schraiber LB, D'oliveira AFPL, Júnior IF, Pinho AA Violência contra a mulher: Estudo em uma unidade de atenção primária à saúde. Rev Saúde Pública 2002; 36(4); 470-7.
15. Brasil. Ministério da Saúde. Secretaria de Vigilância em Saúde. Impacto da violência na saúde dos brasileiros. Brasília, 2005.

16. Borsoi TS, Brandão ER, Cavalcanti, S. Ações para o enfrentamento da violência contra a mulher em duas unidades de atenção primária à saúde no município do Rio de Janeiro - Rio de Janeiro. Interface - Comunic., Saúde, Educ., 2009;13(28):165-74.

17. Oliveira $C$. Violência doméstica na perspectiva de gênero e políticas públicas. Rev Bras Crescimento Desenvolv Hum. 2007;17(1):39-51.

18. Scott J. Gender: a useful category of historical analyses Gender and the politics of history. New York, Columbia University Press. 1989

19. IBAHIA - São Francisco do Conde, na Bahia possui maior PIB do País. Disponível em: http:// www.ibahia.com. Acesso em: 10 de março de 2015.

20. Organização Mundial Da Saúde. Informe mundial sobre violência e saúde. 2002. Disponível em: http:// www.who.int/violence injury prevention/. Acesso em: 18 de maio de 2015.

21. Yeager K, Seid A. Primary care and victims of domestic violence. Primary Care; Clinics in Office Pratice, 2002; 29(1); 125-50.

22. Krasnoff M, Moscati R. Domestic violence screening and referral can be effective. Annals of Emergency Medicine, 2002; 40(5); 485-92.

\section{Correspondência}

Howard Lopes Ribeiro Junior

R. Coronel João de Oliveira,1001, Messejana.

CEP: $60841-820$

Fortaleza - Ceará - Brasil.

howard@unilab.edu.br 